\title{
Collagen Type XI Alpha 1 Expression in Intraductal Papillomas Predicts Malignant Recurrence
}

\author{
Javier Freire, ${ }^{1,2}$ Lucia García-Berbel, ${ }^{3}$ Pilar García-Berbel, ${ }^{1,2}$ Saray Pereda, ${ }^{1,2}$ \\ Ainara Azueta, ${ }^{1,2}$ Pilar García-Arranz, ${ }^{1}$ Ana De Juan, ${ }^{4}$ Alfonso Vega, ${ }^{5}$ Ángela Hens, ${ }^{6}$ \\ Ana Enguita, ${ }^{7}$ Pedro Muñoz-Cacho, ${ }^{8}$ and Javier Gómez-Román ${ }^{1,2}$ \\ ${ }^{1}$ Anatomía Patológica, Hospital Universitario Marqués de Valdecilla, 39008 Santander, Spain \\ ${ }^{2}$ IDIVAL, 39011 Santander, Spain \\ ${ }^{3}$ Ginecología y Obstetricia, Hospital Universitario de Puerto Real, 11510 Puerto Real, Spain \\ ${ }^{4}$ Oncología Médica, Hospital Universitario Marqués de Valdecilla, 39008 Santander, Spain \\ ${ }^{5}$ Radiodiagnóstico, Hospital Universitario Marqués de Valdecilla, 39008 Santander, Spain \\ ${ }^{6}$ Anatomía Patológica, Hospital Universitario de Puerto Real, 11510 Puerto Real, Spain \\ ${ }^{7}$ Anatomía Patológica, Hospital Universitario 12 de Octubre, 28041 Madrid, Spain \\ ${ }^{8}$ Gerencia Atención Primaria, Servicio Cántabro de Salud, 39011 Santander, Spain
}

Correspondence should be addressed to Javier Freire; javifreiresalinas@gmail.com

Received 14 January 2015; Accepted 17 February 2015

Academic Editor: Konstantinos Arnaoutakis

Copyright (C) 2015 Javier Freire et al. This is an open access article distributed under the Creative Commons Attribution License, which permits unrestricted use, distribution, and reproduction in any medium, provided the original work is properly cited.

Despite the progress achieved in the treatment of breast cancer, there are still many unsolved clinical issues, being the diagnosis, prognosis, and treatment of papillary diseases, one of the highest challenges. Because of its unpredictable clinical behavior, treatment of intraductal papilloma has generated a great controversy. Even though considered as a benign lesion, it presents high rate of malignant recurrence. This is the reason why there are clinicians supporting a complete excision of the lesion, while others support an only expectant follow-up. Previous results of our group suggested that procollagen 11 alpha 1 (pro-COL11A1) expression correlates with infiltrating phenotype in breast lesions. We analyzed the correlation between expression of pro-COL11A1 in intraductal papilloma and their risk of malignant recurrence. Immunohistochemistry of pro-COL11A1 was performed in 62 samples of intraductal papilloma. Ten out 11 cases relapsed as carcinoma presents positive staining for COL11A1, while just 17 out of 51 cases with benign behaviour present immunostaining. There were significant differences $(P<0.0001)$ when comparing patients with malignant recurrence versus nonmalignant relapse patients. These data suggest that pro-COL11A1 expression is a highly sensitive biomarker to predict malignant relapse of intraductal papilloma and it can be used as indicative factor for prevention programs.

\section{Background}

Breast cancer is the first tumor disease among women, causing more than 600000 new cases per year [1]. Furthermore it is also the second cause of cancer death among women, causing more than 39,000 deaths each year only in United States [2]. Although in the last years the early detection of this disease has improved overall survival [3], breast cancer remains a very serious problem for public health and there are still open many research areas.

Papillary lesions (intraductal papilloma, papillomatosis, atypical papilloma, and intraductal papillary carcinoma) are controversial and continuously generate problems in diagnosis and clinical management [4]. Because of their similarity, the accurate diagnosis of these lesions only by morphology may be complex, so pathologist requires the use of ancillary techniques. The main indicator of malignancy of papillary lesion is the absence of myoepithelial cells [5] which can be revealed by immunohistochemistry for p63 protein, smooth muscle actin (SMM-HC), or calponin [6]. Other biomarkers have been used as estrogen receptor or cytokeratins [6] CK5/6 and CK8 [7] for differential diagnosis but there is a no clear consensus to determine the sensitivity and accuracy of these markers in routine $[5,8]$. 
Intraductal papilloma is the most controversial papillary lesion relating diagnosis and treatment [6]. While intraductal papilloma per se behaves like a benign lesion, the association between intraductal papilloma and malignant recurrence is fairly high, reaching up to $33 \%$ of the cases $[5,9,10]$. Indeed, there is a great controversy on how to act when a new case of intraductal papilloma is diagnosed. In fact there are papers suggesting a radical excision of the lesion in all cases $[11,12]$, while others support only an expectant follow-up [13-15]. An accurate diagnosis pointing to cases amenable of a malignant behavior is essential $[6,16,17]$, not only for the benefit of the patient, as it would avoid unnecessary interventions, but also because of its economic impact [8].

It has been shown that the extracellular matrix plays an essential role in breast tumor development and progression, being collagens its main component. Collagen type XI alpha 1 (COL11A1) has been shown to be a marker of malignancy in different tumors including pancreas [18], lung [19], stomach [20], and colon [21-23]. Previous work from our group has demonstrated that pro-COL11A1 expression in cancer associated fibroblasts is a powerful marker of invasive growth in breast carcinomas, with sensitivity and specificity rates higher than $90 \%$ [24]. COL11A1 is not present in benign lesions so we thought it can be a predictable marker for malign behavior of intraductal papilloma.

\section{Mat and Meth}

2.1. Tissue Samples. Sixty-two patients with a clinicopathological diagnosis of breast intraductal papilloma from the University Hospital Marqués de Valdecilla (Santander, Spain), University Hospital of Puerto Real (Puerto Real, Spain) and University Hospital 12 de Octubre (Madrid, Spain) were enrolled for this work. All the samples examined were core needle biopsies from $18 \mathrm{G}$ gauge.

Patients were diagnosed by two independent pathologists following the standard work routine. All patients recruited for the study had a minimum follow-up of 5 years. Patient recruitment was conducted under approvement by the Clinical Research Ethics Committee of Cantabria.

Five cases of encapsulated papillary carcinoma were also selected as positive control of malignant lesion.

2.2. Immunohistochemical Analysis. Formalin fixed, paraffin embedded biopsies were stained by using proColl1al monoclonal antibody clone 1E8.33 (ONCOMATRYX, Bilbao, SPAIN) as previously described [24, 25]. Samples were considered as positive when a clear cytoplasmic labeling of at least one tumor-associated fibroblast was observed. Staining was separately evaluated by two independent pathologists.

2.3. Statistical Methods. Nonparametric Fisher exact test was performed, using SPSS 20 suite, to analyze difference of COL11A1 expression between intraductal papilloma with or without malignant relapse. Survival analyses were performed using Kaplan-Meier curves, and hazard ratio (HR) and corresponding 95\% confident interval (95\% CI) were estimated using Cox proportional hazards regression of recurrence for positive staining for COL11A1.

\section{Results}

Out of 62 cases studied, 11 presented recurrence as an infiltrative carcinoma, 7 presented further nonmalignant proliferative lesion (papilloma, columnar hyperplasia...) while 44 remaining cases showed no recurrence. Benign relapsed or no recurrence samples were considered as a single group for comparing with those which presented as a malignant relapse. Immunolabeling of pro-COL11A1 was observed in fibroblasts surrounding central fibrovascular stalks.

Among papillomas with malignant relapse $91 \%$ showed positive staining (Figure 1(b)), whereas those papillomas with benign or not recurrence present only $33 \%$ of immunostaining $(P<0.0001)$ (Figure 1(a)). All five encapsulated papillary carcinoma were positive for COL11A1 staining (Figure $1(\mathrm{C})$ ).

Pro-COL11A1 staining showed sensitivity of $91 \%$ and a specificity of $67 \%$ when compared intraductal papilloma malignant relapsed samples with those not recurrent. Moreover, Cox regression analysis for recurrence risk presents highly statistical significance $(P=0.0008)$ while comparing positive and negative staining, with a HR of 12.6 (3.8-41.4) (Figure 1 sup data) (see Supplementary Material available online at http://dx.doi.org/10.1155/2015/812027).

\section{Discussion}

The present work demonstrates that the presence of COL11A1 in the stroma of breast intraductal papillomas could be a potential marker of malignant behavior.

Breast intraductal papillomas are considered as benign indolent lesions but a significant number of patients are suitable to develop a malignant recurrence [26] something that explain the huge controversy over the treatment to be applied in these kind of lesions [4, 27-30].

Several clinical groups argue for an aggressive complete excisional treatment when an intraductal papilloma is diagnosed, going from a tumorectomy whether solitary papillomas to a radical mastectomy in the case of diffuse lesions $[11,12,31-33]$. On the other hand there are works suggesting the treatment of breast intraductal papilloma to be not so invasive and based in a conservative image-controlled followup $[13-15,34]$. The possibility of making a recommendation for excision only in specific cases where an uncertain degree of malignancy is present is also discussed [35]. This could be a nice approach but, how is the malignancy probability of a pure intraductal papilloma determined [8]? The answer must be coming from morphology and characteristics of neoplastic as well as stromal cells.

Breast intraductal papilloma presents a high rate of underestimation (12-19\%) when it is diagnosed in CoreNeedle Biopsy $[10,29,36]$ mainly due to small sample and to indefinite histopathological features [29]. This is why a reliable system for classifying papillary lesions according to malignant potential is required. 


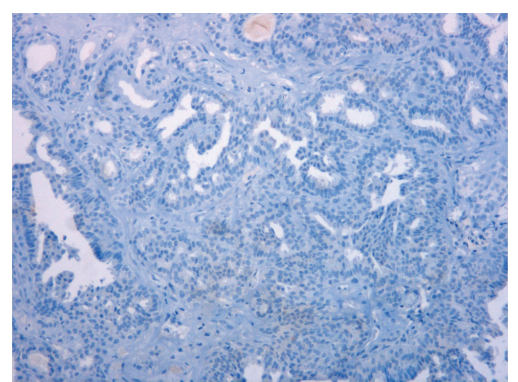

(a)

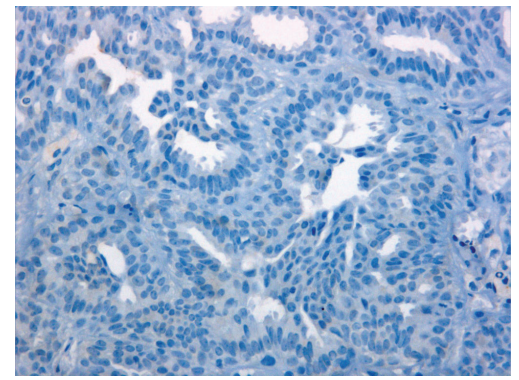

(A)

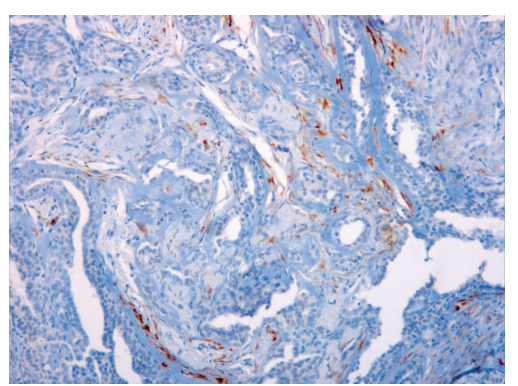

(b)

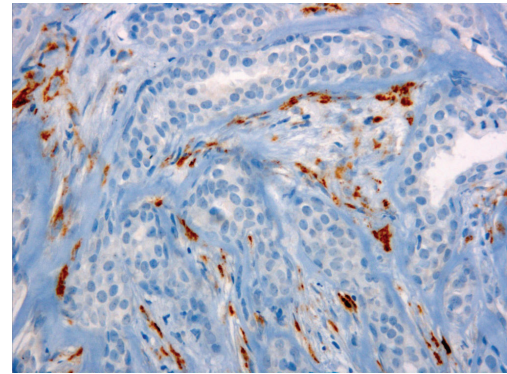

(B)

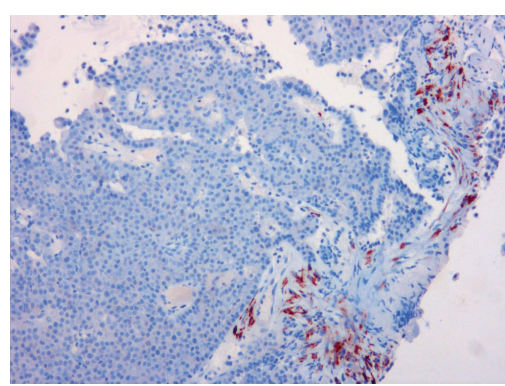

(c)

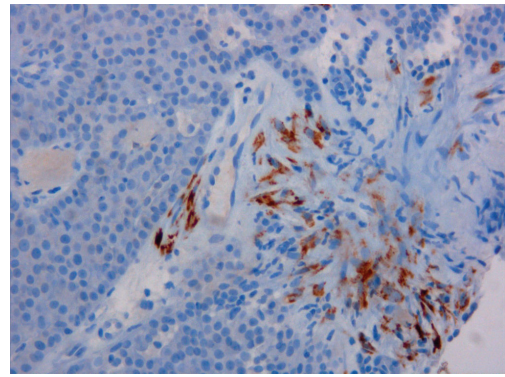

(C)

Figure 1: Pro-COL11A1 expression in breast papillary lesions. Immunostaining for pro-COL11A1 in: (a) benign intraductal papilloma, (b) malignant relapse intraductal papilloma, (c) encapsulated papillary carcinoma. Counterstain with Hematoxilin. Lowercase letters images magnification $\times 200$, uppercase letters images magnification $\times 400$.

Although several biomarkers have been suggested for differentiating potential malignant phenotype of benign intraductal papillomas, none has been demonstrated as an accurate predictive factor of malignancy. Markers as the CD44 [37] or cyclin D1 [38] have been proposed as differentially expressed genes between malignant and benign papillary lesions, but there is no correlation with malignant recurrence of intraductal papillomas. Some genetic alterations, such as loss of heterozygosity of chromosome 16 [39], have also been proposed as capable of predicting an increased susceptibility for malignant recurrence of intraductal papillomas, but accuracy has not been demonstrated [38,39].

Our work is in the cutting edge for classification of intraductal papillomas because it is based on tumor associated fibroblasts and not in the neoplastic cells by itself or in the presence or absence of myoepithelial cells. COL11A1 expression in fibroblast surrounding central fibrovascular stalks of intraductal papillomas can predict future malignant relapse with a sensitivity of $91 \%$. Although the specificity derived from our data is not so high (65\%) it can be explained primarily because the elective treatment for intraductal papillomas in Spain is the complete excision of the lesion, which prevents secondary recurrence.

Positive staining in all encapsulated papillary carcinoma suggests what has been discussed for some time that these lesions, long considered variations of DCIS, may in fact be a form of low-grade invasive carcinoma with an expansile growth pattern [40, 41]. This fact supports our hypothesis of a dual nature of intraductal papillomas: malignant papillary carcinomas or intraductal papillomas with benign prognosis.
This marker combined with other prognostic events such as size larger than $1.5 \mathrm{~cm}$, location [28], or presence of microcalcifications [42] can assist when deciding the possibility of an aggressive treatment versus a conservative follow-up. In any case, the absence of COL11A1 in a biopsy can predict with a high probability that an intraductal papilloma will present a benign behavior since it presents a recurrence HR value of $0.0793(0.02-0.26)$, although changing in therapeutic behavior seems complicated without further studies.

Given that this injury occurs predominantly in pre- and postmenopausal [30] women and that breast intraductal papillary lesions are usually hormone-dependent [43] (in our series more than $85 \%$ estrogens positive), these patients may be susceptible to receive an chemoprevention with hormone inhibitors. It has been demonstrated in different studies that the inhibition of both estrogen receptors (tamoxifen and raloxifene) [44-46] and aromatase pathway (exametasane) [47] reduces contralateral breast cancer relapse. The major problem of these therapies is the election of patients to receive treatment, we propose that COL11Al positive biopsy should be a new factor to ponder besides a Gail 5year risk score greater than $1.66 \%$ and prior preneoplastic lesion [47] to select candidates for this chemoprevention as these lesions have a high susceptibility to malignant relapse.

To conclude, the expression of COL11A1 in breast intraductal papillomas is an optimal prognostic biomarker, and we propose that patients with positive staining for this protein should be given further evaluation of both surgical treatment and preventive adjuvant chemotherapy. 


\section{Conflict of Interests}

The authors declare that there is no conflict of interests regarding the publication of this paper.

\section{Acknowledgments}

The authors are grateful to the nursing unit of radiodiagnosis service for their selfless help in the patient recruitment. This study was supported by grants from the Government of Spain through the INNPACTO program, Project Mamacan IPT 2011-1817-900000.

\section{References}

[1] R. Siegel, D. Naishadham, and A. Jemal, "Cancer statistics, 2012," CA Cancer Journal for Clinicians, vol. 62, no. 1, pp. 10-29, 2012.

[2] C. DeSantis, R. Siegel, P. Bandi, and A. Jemal, "Breast cancer statistics, 2011," CA: A Cancer Journal for Clinicians, vol. 61, no. 6, pp. 409-418, 2011.

[3] M. T. Tirona, "Breast cancer screening update," American Family Physician, vol. 87, no. 4, pp. 274-278, 2013.

[4] L. C. Collins and S. J. Schnitt, "Papillary lesions of the breast: selected diagnostic and management issues," Histopathology, vol. 52, no. 1, pp. 20-29, 2008.

[5] S.-H. Ueng, T. Mezzetti, and F. A. Tavassoli, "Papillary neoplasms of the breast: a review," Archives of Pathology and Laboratory Medicine, vol. 133, no. 6, pp. 893-907, 2009.

[6] A. M. Mulligan and F. P. O'Malley, "Papillary lesions of the breast: a review," Advances in Anatomic Pathology, vol. 14, no. 2, pp. 108-119, 2007.

[7] M. Moumen, A. Chiche, S. Cagnet et al., "The mammary myoepithelial cell," International Journal of Developmental Biology, vol. 55, no. 7-9, pp. 763-771, 2011.

[8] D. Shouhed, F. F. Amersi, R. Spurrier et al., "Intraductal papillary lesions of the breast: clinical and pathological correlation," The American Surgeon, vol. 78, no. 10, pp. 1161-1165, 2012.

[9] P. P. Rosen, "Papilloma and related benign tumors," in Rosen's Breast Pathology, P. P. Rosen, Ed., pp. 85-136, Lippincott Williams \& Wilkins, Philadelphia, Pa, USA, 2009.

[10] E. K. Valdes, P. I. Tartter, E. Genelus-Dominique, D.-A. Guilbaud, S. Rosenbaum-Smith, and A. Estabrook, "Significance of papillary lesions at percutaneous breast biopsy," Annals of Surgical Oncology, vol. 13, no. 4, pp. 480-482, 2006.

[11] M. Rizzo, M. J. Lund, G. Oprea, M. Schniederjan, W. C. Wood, and M. Mosunjac, "Surgical follow-up and clinical presentation of 142 breast papillary lesions diagnosed by ultrasound-guided core-needle biopsy," Annals of Surgical Oncology, vol. 15, no. 4, pp. 1040-1047, 2008.

[12] A. R. Skandarajah, L. Field, A. Yuen Larn Mou et al., "Benign papilloma on core biopsy requires surgical excision," Annals of Surgical Oncology, vol. 15, no. 8, pp. 2272-2277, 2008.

[13] S. N. Agoff and T. J. Lawton, "Papillary lesions of the breast with and without atypical ductal hyperplasia: can we accurately predict benign behavior from core needle biopsy," The American Journal of Clinical Pathology, vol. 122, no. 3, pp. 440-443, 2004.

[14] E. L. Rosen, R. C. Bentley, J. A. Baker, and M. S. Soo, "Imagingguided core needle biopsy of papillary lesions of the breast," American Journal of Roentgenology, vol. 179, no. 5, pp. 1185-1192, 2002.
[15] V. Sohn, J. Keylock, Z. Arthurs et al., "Breast papillomas in the era of percutaneous needle biopsy," Annals of Surgical Oncology, vol. 14, no. 10, pp. 2979-2984, 2007.

[16] Y. D. Choi, G. Y. Gong, M. J. Kim et al., "Clinical and cytologic features of papillary neoplasms of the breast," Acta Cytologica, vol. 50, no. 1, pp. 35-40, 2006.

[17] V. Gomez-Aracil, E. Mayayo, J. Azua, and A. Arraiza, "Papillary neoplasms of the breast: clues in fine needle aspiration cytology," Cytopathology, vol. 13, no. 1, pp. 22-30, 2002.

[18] M. Erkan, N. Weis, Z. Pan et al., "Organ-, inflammation- and cancer specific transcriptional fingerprints of pancreatic and hepatic stellate cells," Molecular Cancer, vol. 9, article 88, 2010.

[19] I.-W. Chong, M.-Y. Chang, H.-C. Chang et al., "Great potential of a panel of multiple hMTH1, SPD, ITGA11 and COL11A1 markers for diagnosis of patients with non-small cell lung cancer," Oncology Reports, vol. 16, no. 5, pp. 981-988, 2006.

[20] Y. Zhao, T. Zhou, A. Li et al., "A potential role of collagens expression in distinguishing between premalignant and malignant lesions in stomach," Anatomical Record (Hoboken), vol. 292, no. 5, pp. 692-700, 2009.

[21] K. B. Bowen, A. P. Reimers, S. Luman, J. D. Kronz, W. E. Fyffe, and J. Thom, "Immunohistochemical localization of collagen type XI $\alpha 1$ and $\alpha 2$ chains in human colon tissue," Journal of Histochemistry \& Cytochemistry, vol. 56, no. 3, pp. 275-283, 2008.

[22] H. Fischer, S. Salahshor, R. Stenling et al., "COL11A1 in FAP polyps and in sporadic colorectal tumors," BMC Cancer, vol. 1, article 17, 2001.

[23] H. Fischer, R. Stenling, C. Rubio, and A. Lindblom, "Colorectal carcinogenesis is associated with stromal expression of COL11A1 and COL5A2," Carcinogenesis, vol. 22, no. 6, pp. 875$878,2001$.

[24] J. Freire, "Collagen, type XI, alpha 1: an accurate marker for differential diagnosis of breast carcinoma invasiveness in core needle biopsies," Pathology-Research and Practice, vol. 210, no. 12, pp. 879-884, 2014.

[25] M. García-Ocaña, F. Vázquez, C. García-Pravia et al., "Characterization of a novel mouse monoclonal antibody, clone 1E8.33, highly specific for human procollagen 11A1, a tumor-associated stromal component," International Journal of Oncology, vol. 40, no. 5, pp. 1447-1454, 2012.

[26] J. A. Ibarra, "Papillary lesions of the breast," Breast Journal, vol. 12, no. 3, pp. 237-251, 2006.

[27] L. E. Bennett, S. V. Ghate, R. Bentley, and J. A. Baker, "Is surgical excision of core biopsy proven benign papillomas of the breast necessary?" Academic Radiology, vol. 17, no. 5, pp. 553-557, 2010.

[28] W.-H. Kil, E. Y. Cho, J. H. Kim, S.-J. Nam, and J.-H. Yang, "Is surgical excision necessary in benign papillary lesions initially diagnosed at core biopsy?" Breast, vol. 17, no. 3, pp. 258-262, 2008.

[29] Q. Lu, E. Y. Tan, B. Ho, J. J. C. Chen, and P. M. Y. Chan, "Surgical excision of intraductal breast papilloma diagnosed on core biopsy," ANZ Journal of Surgery, vol. 82, no. 3, pp. 168-172, 2012.

[30] T. C. Putti, S. E. Pinder, C. W. Elston, A. H. S. Lee, and I. O. Ellis, "Breast pathology practice: most common problems in a consultation service," Histopathology, vol. 47, no. 5, pp. 445-457, 2005.

[31] K. Irfan and R. F. Brem, "Surgical and mammographic followup of papillary lesions and atypical lobular hyperplasia diagnosed with stereotactic vacuum-assisted biopsy," Breast Journal, vol. 8, no. 4, pp. 230-233, 2002. 
[32] T. W. Jacobs, J. L. Connolly, and S. J. Schnitt, "Nonmalignant lesions in breast core needle biopsies: to excise or not to excise?" The American Journal of Surgical Pathology, vol. 26, no. 9, pp. 1095-1110, 2002.

[33] F. Puglisi, C. Zuiani, M. Bazzocchi et al., "Role of mammography, ultrasound and large core biopsy in the diagnostic evaluation of papillary breast lesions," Oncology, vol. 65, no. 4, pp. 311-315, 2003.

[34] N. Ahmadiyeh, M. A. Stoleru, S. Raza, S. C. Lester, and M. Golshan, "Management of intraductal papillomas of the breast: an analysis of 129 cases and their outcome," Annals of Surgical Oncology, vol. 16, no. 8, pp. 2264-2269, 2009.

[35] P. J. Carder, J. Garvican, I. Haigh, and J. C. Liston, "Needle core biopsy can reliably distinguish between benign and malignant papillary lesions of the breast," Histopathology, vol. 46, no. 3, pp. 320-327, 2005.

[36] X. Wen and W. Cheng, "Nonmalignant breast papillary lesions at core-needle biopsy: a meta-analysis of underestimation and influencing factors," Annals of Surgical Oncology, vol. 20, no. 1, pp. 94-101, 2013.

[37] G. M. K. Tse, P.-H. Tan, T. K. F. Ma, C. B. Gilks, C. S. P. Poon, and B. K. B. Law, "CD44s is useful in the differentiation of benign and malignant papillary lesions of the breast," Journal of Clinical Pathology, vol. 58, no. 11, pp. 1185-1188, 2005.

[38] M. Saddik, R. Lai, L. J. Medeiros, A. McCourty, and R. K. Brynes, "Differential expression of cyclin D1 in breast papillary carcinomas and benign papillomas: an immunohistochemical study," Archives of Pathology and Laboratory Medicine, vol. 123, no. 2, pp. 152-156, 1999.

[39] M. Yoshida, H. Tsuda, S. Yamamoto et al., "Loss of heterozygosity on chromosome $16 \mathrm{q}$ suggests malignancy in core needle biopsy specimens of intraductal papillary breast lesions," Virchows Archiv, vol. 460, no. 5, pp. 497-504, 2012.

[40] C. A. Wynveen, T. Nehhozina, M. Akram et al., "Intracystic papillary carcinoma of the breast: an in situ or invasive tumor? results of immunohistochemical analysis and clinical followup," American Journal of Surgical Pathology, vol. 35, no. 1, pp. 1-14, 2011.

[41] E. A. Rakha, N. Gandhi, F. Climent et al., "Encapsulated papillary carcinoma of the breast: an invasive tumor with excellent prognosis," The American Journal of Surgical Pathology, vol. 35, no. 8, pp. 1093-1103, 2011.

[42] X. Li, O. Weaver, M. M. Desouki et al., "Microcalcification is an important factor in the management of breast intraductal papillomas diagnosed on core biopsy," The American Journal of Clinical Pathology, vol. 138, no. 6, pp. 789-795, 2012.

[43] F. O’Malley, D. Visscher, G. MacGrogan, P. H. Tan, and S. Ichihara, "Intraductal papilloma," in WHO Classification of Tumours of the Breast, S. R. Lakhani, I. O. Ellis, S. J. Schnitt, P. H. Tan, and M. J. van de Vijver, Eds., pp. 99-105, International Agency of Research on Cancer (IARC), Lyon, France, 2012.

[44] J. Cuzick, T. Powles, U. Veronesi et al., "Overview of the main outcomes in breast-cancer prevention trials," The Lancet, vol. 361, no. 9354, pp. 296-300, 2003.

[45] B. Fisher, J. P. Costantino, D. L. Wickerham et al., "Tamoxifen for the prevention of breast cancer: current status of the National Surgical Adjuvant Breast and Bowel Project P-1 study," Journal of the National Cancer Institute, vol. 97, no. 22, pp. 16521662, 2005.

[46] V. G. Vogel, J. P. Costantino, D. L. Wickerham et al., "Effects of tamoxifen vs raloxifene on the risk of developing invasive breast cancer and other disease outcomes: the NSABP study of tamoxifen and raloxifene (STAR) P-2 trial," The Journal of the American Medical Association, vol. 295, no. 23, pp. 2727-2741, 2006.

[47] P. E. Goss, J. N. Ingle, J. Ales-Martinez et al., "Exemestane for breast-cancer prevention in postmenopausal women," The New England Journal of Medicine, vol. 364, no. 25, pp. 2381-2391, 2011. 


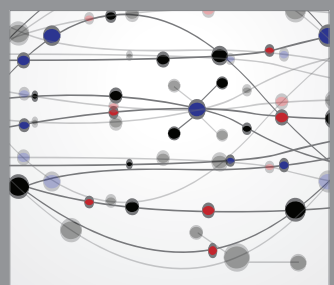

The Scientific World Journal
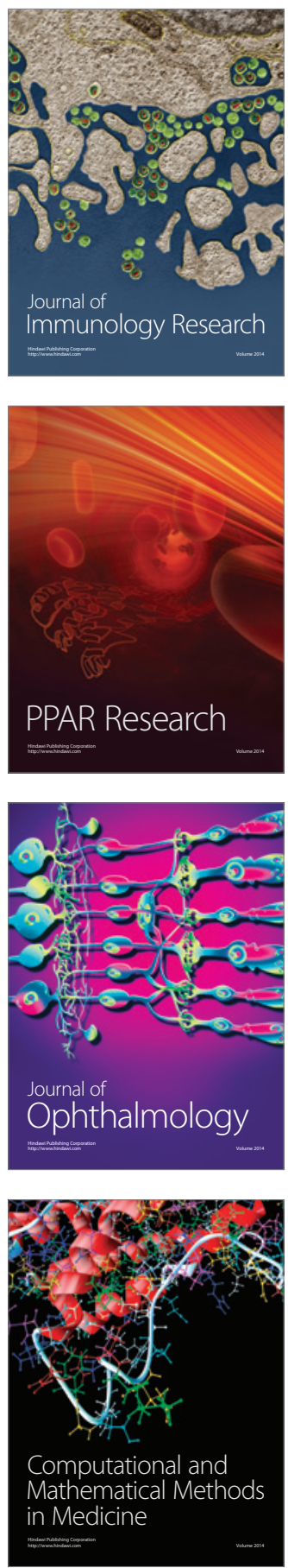

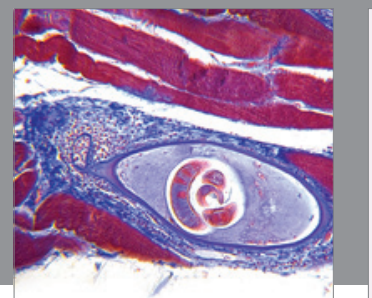

Gastroenterology

Research and Practice
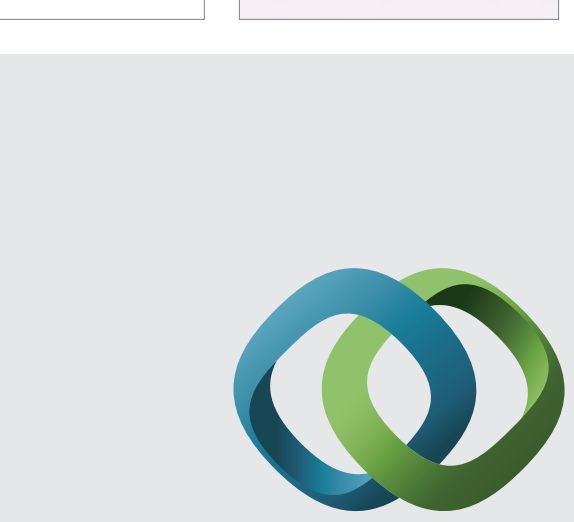

\section{Hindawi}

Submit your manuscripts at

http://www.hindawi.com
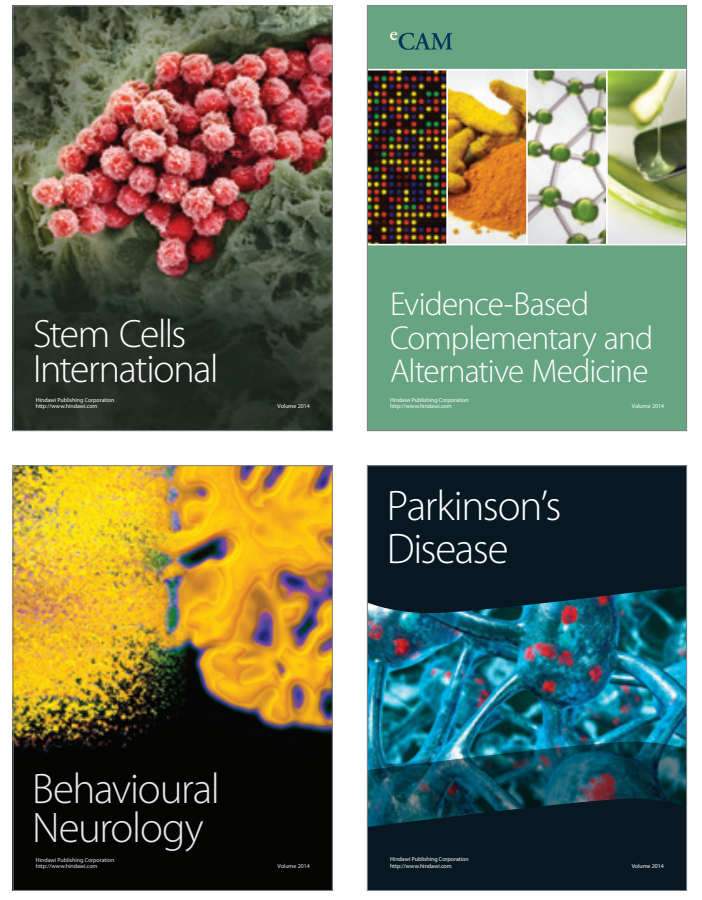
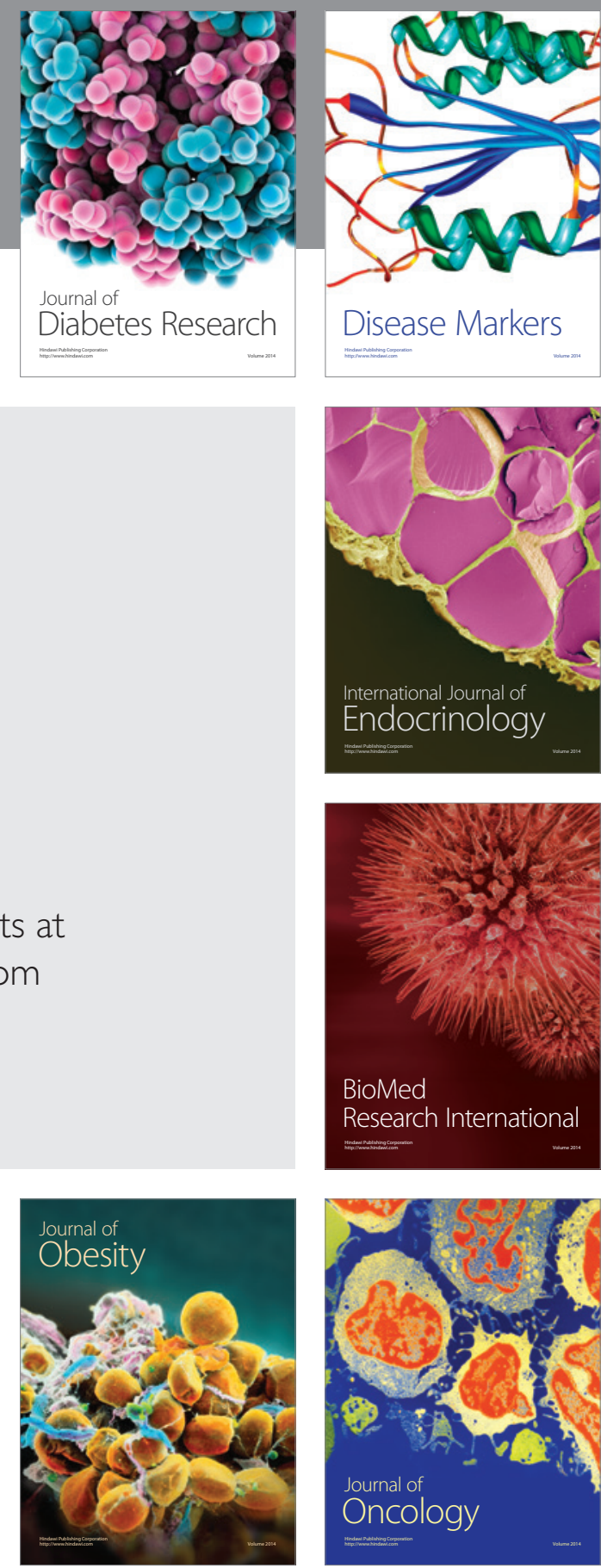

Disease Markers
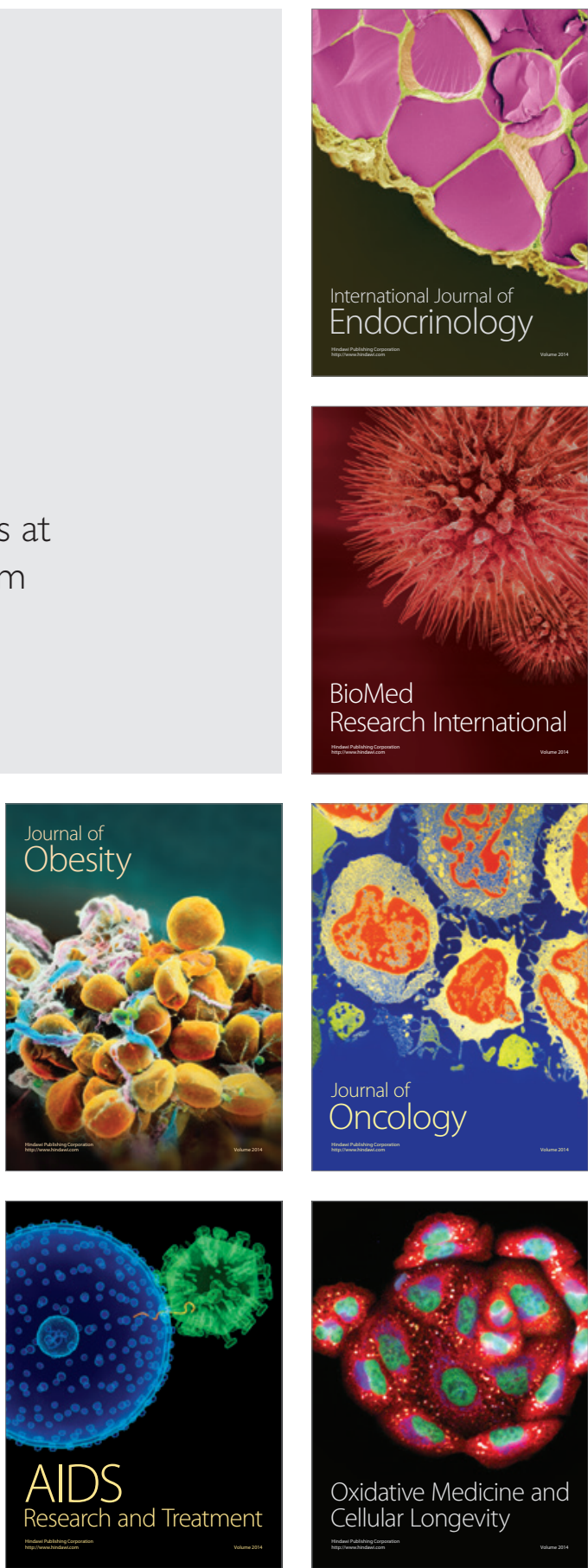